\title{
ON THE $(s, s)$ POLICY WITH FIXED INVENTORY HOLDING AND SHORTAGE COSTS
}

\author{
Tomonori Ishigaki \\ Nagoya Institute of Technology \\ Katsushige Sawaki \\ Nanzan University
}

(Received February 20, 1989; Final August 13, 1990)

\begin{abstract}
In this paper, we consider a dynamic stochastic inventory model with fixed inventory holding and shortage costs in addition to a fixed ordering cost. We discuss a sufficient and necessary condition for an $(s, S)$ policy to be optimal in the class of such stochastic inventory models. Furthermore, we explore how such a sufficient and necessary condition can be rewritten when the demand distribution is specified. Several examples such as uniform, exponential, normal and gamma distribution functions are treated. The main purpose of this paper is to show that the $(s, S)$ policy is still optimal under a simple condition even if fixed inventory costs are involved. Although Aneja and Noori [1] consider a similar model only with fixed inventory shortage cost, our proof for the optimality of an $(s, S)$ policy in the multi-period model is different from and much simpler than theirs.
\end{abstract}

\section{Introduction}

It is well known (see Scarf[7] and Veinott[8] [9] [10])that an $(\mathrm{s}, \mathrm{S})$ policy is optimal for the stochastic inventory control problem with fixed and proportional production costs. As to dynamic stochastic inventory control, the concept of $K$-convexity is crucial for the discussion of an optimal policy which is an $(\mathrm{s}, \mathrm{S})$ type. However, if the inventory cost includes a fixed cost, the $(\mathrm{s}, \mathrm{S})$ policy is no longer optimal. For example, Aneja and Noori[1] discuss a sufficient condition for the $(\mathrm{s}, \mathrm{S})$ policy to be optimal if the inventory shortage cost has a fixed part but the inventory holding cost is not fixed.

In this paper, we shall discuss the relationship between the optimality of an $(\mathrm{s}, \mathrm{S})$ policy and the fixed inventory holding and shortage costs, in addition to the production cost with fixed cost. Our model is not only an extension of Aneja and Noori[1], but provides a different and simpler proof for the optimality of an optimal $(\mathrm{s}, \mathrm{S})$ policy for the dynamic stochastic inventory problem. Also, this paper aims to answer the following question: What is a sufficient condition for the $(\mathrm{s}, \mathrm{S})$ policy to be optimal if there is a fixed inventory cost? In other words, how robust is the $(\mathrm{s}, \mathrm{S})$ policy with respect to the inventory cost function? Furthermore, we analyze how such a sufficient condition can be rewritten and whether it holds or not when the demand distribution is specified, such as in the case of uniform, exponential, normal or gamma distributions.

\section{Preliminaries}

In this paper, we consider a finite period dynamic stochastic inventory problem with a single item. We require the following assumptions and notations:

- The unsatisfied demand is lost.

- If the demand is less than the stock level, then holding cost incurs at the end of each period. This holding cost consists of two parts, the fixed holding cost $\left[B_{1}\right]$ and the proportional holding cost $[h]$. 
- If the demand is bigger than the stock level, then shortage cost incurs. This shortage cost again consists of two parts, the fixed shortage cost $\left[B_{2}\right]$ and the proportional shortage cost $[p]$.

- If an order is taken, then the ordering cost incurs. This ordering cost consists of the fixed ordering cost $[K]$ and the proportional cost $[c]$.

- The demand of each period is given by the random variable $[\xi]$ which has the probability density function (p.d.f.) $\phi(\xi)$. We assume that p.d.f. $\phi(\xi)$ is differentiable.

- Both the cost functions and the p.d.f. of demand are identical over the periods.

Let us assume that the planning horizon is discrete and finite, and consists of $N$ periods. First, we consider the expected cost over $n$ periods $(n \leq N)$. If the stock level immediately after an ordering is $y$, then the sum of the expected holding and shortage costs to be charged during a period is given by

$$
L(y)=h \int_{0}^{y}(y-\xi) \phi(\xi) d \xi+B_{1} \int_{0}^{y} \phi(\xi) d \xi+p \int_{y}^{\infty}(\xi-y) \phi(\xi) d \xi+B_{2} \int_{y}^{\infty} \phi(\xi) d \xi
$$

where we assume that $B_{2}$ is not equal to $B_{1}$. If $B_{1}=B_{2}$, then it is easy to see from equation(2.1) that the sum of the fixed holding and shortage costs is independent of $y$. Therefore, this model reduces to the classical stochastic inventory model with the only fixed cost being a fixed ordering cost. Let $C_{n}(x)$ be the minimum of the expected total discounted cost over $n$-periods when $x$ is the starting inventory level before an ordering at the beginning of period $n$. Then, we have from the principle of the optimality

$$
C_{n}(x)=\min _{y \geq x}\left\{H(y-x)+L(y)+\rho \int_{0}^{\infty} C_{n-1}\left([y-\xi]^{+}\right) \phi(\xi) d \xi\right\}
$$

where $[y]^{+}=\max \{y, 0\}, n=1,2, \ldots, N, \rho$ is the discount factor, $0<\rho \leq 1, C_{0}(x)=0$ for all $x$ and $H(\cdot)$ is defined as follows;

$$
H(y-x)= \begin{cases}0, & \text { if } y-x \leq 0 \\ K+c \cdot(y-x), & \text { otherwise }\end{cases}
$$

The objective of this model is to find an optimal inventory policy which minimizes the expected total discounted cost. To prove the optimality of an $(\mathrm{s}, \mathrm{S})$ policy for the multiperiod model, we first consider the single-period model of this problem.

\section{Single-Period Model}

In this section, we discuss the optimality of the $(\mathrm{s}, \mathrm{S})$ policy in a single-period model. For $N=1$, equation(2.2) reduces to

$$
C_{1}(x)=\min _{y \geq x}\{H(y-x)+L(y)\}
$$

Theorem 1. A sufficient and necessary condition for the optimality of an $(s, S)$ policy for the single-period problem is that

Condition (A)

$$
\frac{\phi^{\prime}(y)}{\phi(y)}\left\{\begin{array}{l}
\leq \\
\geq
\end{array}\right\} \frac{h+p}{B_{2}-B_{1}} \quad \text { for all } \quad y \in \Re^{+} \quad \text { if } B_{2}-B_{1}\left\{\begin{array}{l}
> \\
<
\end{array}\right\} 0
$$


where $\Re_{+}=\{y \mid y \geq 0\}$.

Proof: (Sufficiency) It suffices to prove for the case, $B_{2}-B_{1}<0$, because the proof of the case, $B_{2}-B_{1}>0$, can be applied to Aneja and Noori's result as $B \equiv B_{2}-B_{1}$. Let $F_{1}(y)$ be the quantity inside the braces of the right-hand-side of equation(3.1) and put $G_{1}(y)=F_{1}(y)-K+c x$. For $y>x$, we have

$$
G_{1}(y)=c y+h \int_{0}^{y}(y-\xi) \phi(\xi) d \xi+B_{1} \int_{0}^{y} \phi(\xi) d \xi+p \int_{y}^{\infty}(\xi-y) \phi(\xi) d \xi+B_{2} \int_{y}^{\infty} \phi(\xi) d \xi .
$$

In this case, the first and second derivatives of the function $G_{1}(y)$ are

$$
G_{1}^{\prime}(y)=c+h \int_{0}^{y} \phi(\xi) d \xi+B_{1} \phi(y)-p \int_{y}^{\infty} \phi(\xi) d \xi-B_{2} \phi(y)
$$

and

$$
\begin{aligned}
G_{1}^{\prime \prime}(y) & =h \phi(y)+B_{1} \phi^{\prime}(y)+p \phi(y)-B_{2} \phi^{\prime}(y) \\
& =(h+p) \phi(y)-\left(B_{2}-B_{1}\right) \phi^{\prime}(y) .
\end{aligned}
$$

From condition (A), we obtain

$$
G_{1}^{\prime \prime}(y)=(h+p) \phi(y)-\left(B_{2}-B_{1}\right) \phi^{\prime}(y) \geq 0,
$$

which concludes that $G_{1}(y)$ is convex in $y$.

If $y=x$, then we have $G_{1}(x)-c x=F_{1}(x)$.

$$
G_{1}(x)=c x+h \int_{0}^{x}(x-\xi) \phi(\xi) d \xi+B_{1} \int_{0}^{x} \phi(\xi) d \xi+p \int_{x}^{\infty}(\xi-x) \phi(\xi) d \xi+B_{2} \int_{x}^{\infty} \phi(\xi) d \xi .
$$

The first and second derivatives of equation(3.7) are

$$
G_{1}^{\prime}(x)=c+h \int_{0}^{x} \phi(\xi) d \xi+B_{1} \phi(x)-p \int_{x}^{\infty} \phi(\xi) d \xi-B_{2} \phi(x)
$$

and

$$
\begin{aligned}
G_{1}^{\prime \prime}(x) & =h \phi(x)+B_{1} \phi^{\prime}(x)+p \phi(x)-B_{2} \phi^{\prime}(x) \\
& =(h+p) \phi(x)-\left(B_{2}-B_{1}\right) \phi^{\prime}(x) .
\end{aligned}
$$

Since this equation is identical with equation(3.6), equations (3.6) and (3.9) imply that $G_{1}(y)$ is a convex function of $y \geq x$.

Therefore,

$$
F_{1}(y)= \begin{cases}G_{1}(y)+K-c x, & \text { if } y>x \\ G_{1}(x)-c x, & \text { if } y=x\end{cases}
$$

Here, the minimum value function $C_{1}(x)$ should be given by

$$
C_{1}(x)= \begin{cases}G_{1}(S)+K-c x=G_{1}(s)-c x, & \text { if } x<s \\ G_{1}(x)-c x, & \text { if } x \geq s\end{cases}
$$

where

$$
\begin{gathered}
S=\arg \min \left\{G_{1}(y)\right\} \\
s=\min \left\{z \mid G_{1}(S)+K=G_{1}(z)\right\} .
\end{gathered}
$$

Consequently, under the condition (A) an optimal inventory policy is as follows; 
1. if $x<s$, order $(S-x)$,

2. if $x \geq s$, do not order.

Such a policy is called the $(\mathrm{s}, \mathrm{S})$ policy.

(Necessity) Suppose that condition (A) does not hold; that is, there exists some $y \in \Re^{+}$ such that

$$
\frac{\phi^{\prime}(y)}{\phi(y)}\left\{\begin{array}{l}
> \\
<
\end{array}\right\} \frac{h+p}{B_{2}-B_{1}} \quad \text { if } \quad B_{2}-B_{1}\left\{\begin{array}{l}
> \\
<
\end{array}\right\} 0 .
$$

Then, we shall show that there are values of parameters $K$ and $c$ for which any $(\mathrm{s}, \mathrm{S})$ policy cannot be optimal. We shall prove it for the case of $B_{2}-B_{1}>0$. The proof for the case $B_{2}-B_{1}<0$ is similar and hence omitted. We can find some $y$ large enough such that

$$
\phi^{\prime}(y)<0
$$

where $\phi(y)$ is assumed to be continuously differentiable. Because $\phi^{\prime}(y)$ is continuous and negative for sufficiently large $y$, there exists $y_{0}$ such that

$$
\frac{\phi^{\prime}\left(y_{0}\right)}{\phi\left(y_{0}\right)}=\frac{h+p}{B_{2}-B_{1}}
$$

In the case that there are several $y_{0}$ 's for which this might be true, let $y_{0}$ be the largest value satisfying(3.16). For all $y$ with $y \geq y_{0}$, an inequality

$$
\frac{\phi^{\prime}(y)}{\phi(y)} \leq \frac{h+p}{B_{2}-B_{1}}
$$

holds, which implies that

$$
G_{1}^{\prime \prime}(y) \geq 0
$$

On the other hand, in the left neighborhood of $y_{0}$, we have

$$
G_{1}^{\prime \prime}(y) \leq 0 \quad \text { for } y \in\left(y_{0}-\delta, y_{0}\right), \delta>0 .
$$

Now, consider the function $f(y)=G_{1}^{\prime}(y)-(c-p)$ which can be rewritten as

$$
f(y)=(h+p) \Phi(y)-\left(B_{2}-B_{1}\right) \phi(y),
$$

where $\Phi$ is the cumulative distribution function(c.d.f.) of $\phi$. Since $f^{\prime}(y)=G_{1}^{\prime \prime}(y)$, it follows from (3.18) and (3.19) that $f(y)$ attains a local minimum at $y_{0}$. (Assuming that this minimum is also global, a shape of $f(y)$ is as shown in Figure 1). By an appropriate choice of $c$, we can ensure that $G_{1}^{\prime}(y)=f(y)+(c-p)=0$ at $y_{1}$ and $y_{2}$ such that $G_{1}^{\prime \prime}\left(y_{1}\right)<0$ and $G_{1}^{\prime \prime}\left(y_{2}\right)>0$. Thus, the function $G_{1}^{\prime}(y)$ has at least two consecutive zeros, one at $y_{1}$ where $G_{1}(y)$ is concave and the other at $y_{2}$ where $G_{1}(y)$ is convex and there are no zeros beyond $y_{2}$. Thus, $G_{1}(y)$ assumes a local maximum at $y_{1}$ and a local minimum at $y_{2}$. Therefore, we can choose an appropriate $K<G_{1}\left(y_{1}\right)-G_{1}\left(y_{2}\right)$. Summing up the above argument, we obtain the optimal inventory policy as follows:

1. order $\left(y_{2}-x\right)$, if $a \leq x \leq b$, 


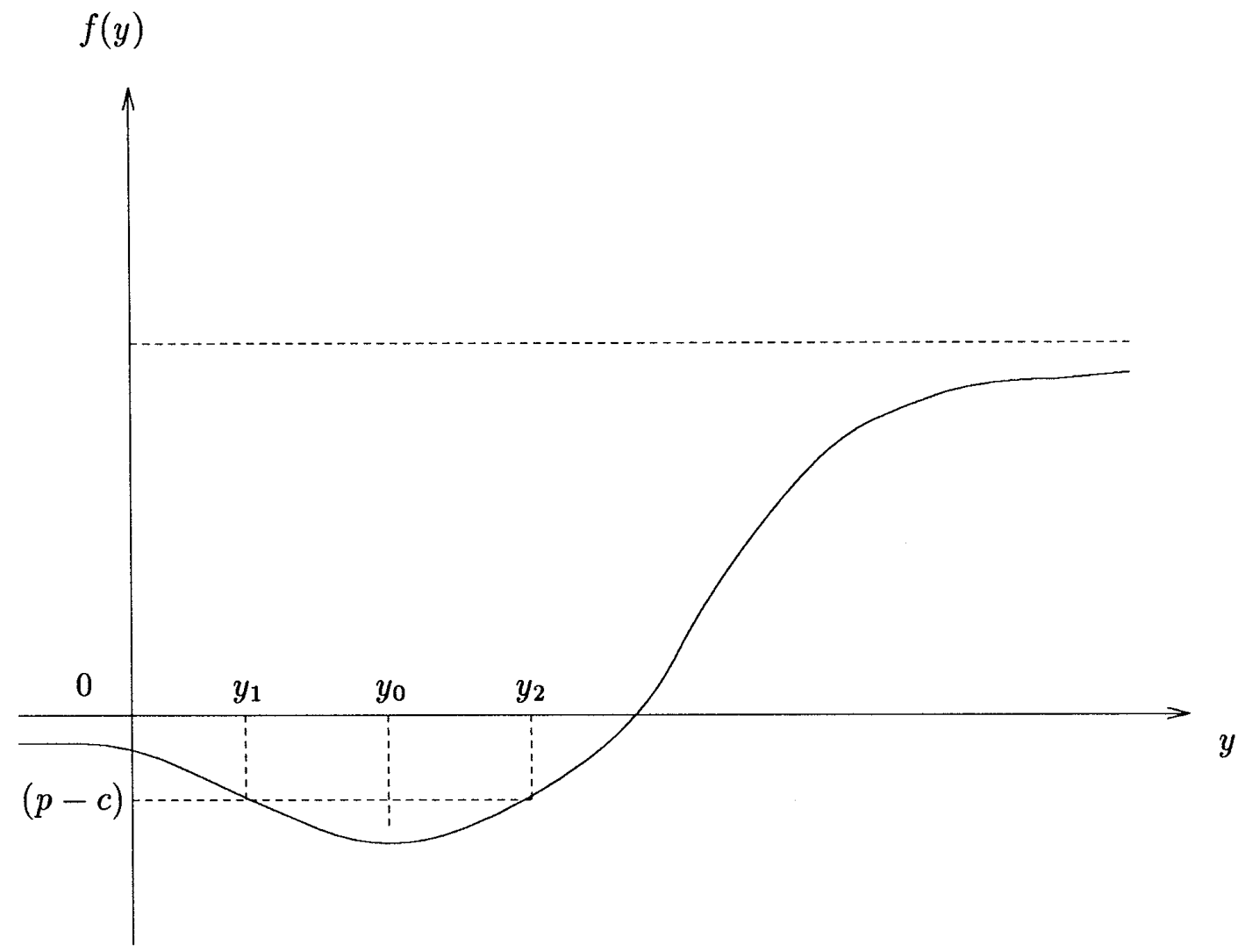

Figure 1: Shape of the function $f(y)$

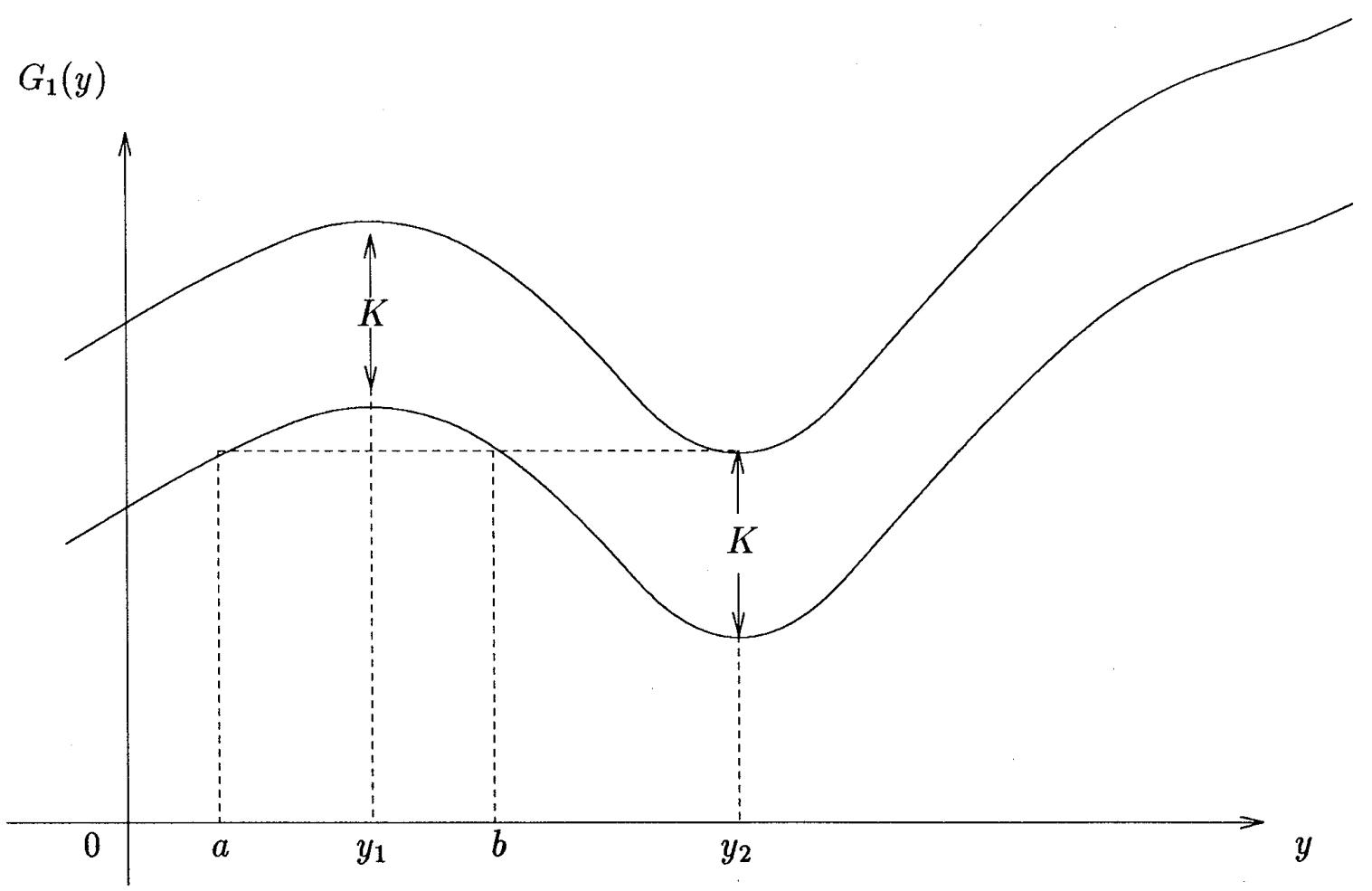

Figure 2: The expected one period cost function 
2. do not order, otherwise,

which is no longer an (s,S) policy(see Figure 2). Thus, condition (A) is necessary for the $(\mathrm{s}, \mathrm{S})$ policy to be optimal.

Remark: Theorem 1 concludes that condition $(A)$ is a sufficient and necessary condition for the $(s, S)$ policy to be optimal. If the right-hand-side of condition $(A)$ is positive, condition (A) holds for all nonincreasing p.d.f. Furthermore, note that our model includes Aneja and Noori[1] type $\left(B_{2}=B, B_{1}=0\right)$ and Scarf[7] type $\left(B_{2}=B_{1}=0\right)$.

\section{Multi-Period Model}

In this section, we shall show that condition (A) is also sufficient for the $(\mathrm{s}, \mathrm{S})$ policy to be optimal in the multi-period model. This is not true in Aneja and Noori[1] because their proof is different from ours. Define $G_{n}(y)$ by

$$
G_{n}(y)=c y+L(y)+\rho \int_{0}^{\infty} C_{n-1}\left([y-\xi]^{+}\right) \phi(\xi) d \xi \quad(y>x)
$$

where $n=1, \ldots, N$. We shall prove Theorem 2 by using properties of a $K$-convex function which is defined as follows;

Definition(K-convexity[7]). Let $K \geq 0$, and let $G_{n}(x)$ be a differentiable function. We say that $G_{n}(x)$ is $K$-convex if

$$
K+G_{n}(a+x)-G_{n}(x)-a G_{n}^{\prime}(x) \geq 0,
$$

for all positive $a$ and all $x$ and $n$.

Before presenting Theorem 2, we require Propositions 1 and 2 whose proofs can be found in the respective references.

\section{Proposition 1(Scarf[7]).}

1. 0-convex function is ordinary convex.

2. If $f(x)$ is $K$-convex, then $f(x+h)$ is $K$-convex for all $h$.

3. If $f$ and $g$ are $K_{1}$-convex and $K_{2}$-convex, respectively, then $(\alpha f+\beta g)$ is $\left(\alpha K_{1}+\beta K_{2}\right)$-convex for $\alpha$ and $\beta$ positive.

4. If $g_{n}(x)$ is $K$-convex, so is $\int_{0}^{\infty} g_{n}(x-\xi) \phi(\xi) d \xi$.

Proposition 2(Denardo[2]). Let $h(y)$ be convex and nondecreasing on $Y$. Let $C(x)$ be $K$-convex on a set $X \supseteq\{h(y) \mid y \in Y\}$. If all elements $a<c$ of $X$ have $C(a) \leq C(c)+K$, then $C[h(y)]$ is $K$-convex on $Y$.

Theorem 2. If condition (A) holds, then $G_{n}(y)$ is $K$-convex in y for each $n$. 
Proof: The Proof is by induction on $n$. For $n=1$, we have $G_{1}(y)=c y+L(y)$ which is convex under condition (A), as we have seen in section 3. Following Scarf's argument (or from equation (3.11)), $C_{1}(x)$ is $K$-convex. Assume that $C_{n-1}(\cdot)$ is $K$-convex. Define

$$
F_{n}(y \mid x) \equiv H(y-x)+L(y)+\rho \int_{0}^{\infty} C_{n-1}\left([y-\xi]^{+}\right) \phi(\xi) d \xi
$$

and put

$$
G_{n}(y)= \begin{cases}F_{n}(y \mid x)-K+c x, & \text { if } y>x \\ F_{n}(x \mid x)+c x, & \text { if } y=x\end{cases}
$$

For $y \geq x$

$$
G_{n}(y)=c y+L(y)+\rho \int_{0}^{\infty} C_{n-1}\left([y-\xi]^{+}\right) \phi(\xi) d \xi .
$$

Since the sum of the first two terms is $G_{1}(y)$, it is 0 -convex under condition (A). The $K$-convexity of the third term can be derived as follows; in a context of proposition 2 , take $C(x)=C_{n-1}(x)$ and $h(y)=[y-\xi]^{+}$. Since $C_{n-1}(x)$ is $K$-convex, then $C_{n-1}(a)-$ $C_{n-1}(c) \leq K$ for $a<c \leq S$, or $a<S<c$. Since $C_{n-1}(x)$ is nondecreasing in $x \geq S$, then $C_{n-1}(a) \leq C_{n-1}(c)+K$ for all $S<a<c$. On the other hand, $h(y)=[y-\xi]^{+}$is nondecreasing and convex in $y$. Hence, the composite function $C_{n-1}\left([y-\xi]^{+}\right)$is $K$-convex in $y$. Therefore, it is easy to see from a simple version of proposition 1-4 that

$$
\rho \int_{0}^{\infty} C_{n-1}\left([y-\xi]^{+}\right) \phi(\xi) d \xi
$$

is $\rho K$-convex. By definition of $K$-convexity, $\rho K$-convexity is $K$-convexity for $0<\rho \leq$ 1. Since $G_{n}(y)$ is the sum of 0 -convex and $K$-convex functions, it is $K$-convex from proposition 1-3.

Theorem 3. If the p.d.f. of demand, $\phi(\xi)$, satisfies condition $(A)$, then an $\left(s_{n}, S_{n}\right)$ policy is optimal for our multi-period inventory problem.

Proof: $\quad$ From theorem 2, we have established $G_{n}(y)$ is $K$-convex for all $n$ and hence, following exactly Scarf's classical arguments, the optimal policy for the $n$-period problem is $\left(s_{n}, S_{n}\right)$ where:

$$
G_{n}\left(S_{n}\right)=\min _{y} G_{n}(y) \quad \text { and } \quad G_{n}\left(s_{n}\right)=K+G_{n}\left(S_{n}\right) .
$$

This policy states that when inventory on hand is below the reorder point $s_{n}$, sufficient stock is ordered to raise the inventory level to the order-up-to-level $S_{n}$ and should not order, otherwise. The minimum expected total cost of following such a policy would be

$$
C_{n}(x)= \begin{cases}K+c\left(S_{n}-x\right)+C_{n}\left(S_{n}\right)=K-c x+G_{n}\left(S_{n}\right), & \text { if } x<s_{n} \\ -c x+G_{n}(x), & \text { if } x \geq s_{n}\end{cases}
$$


Table 1: Left-hand-side of the Condition (A)

\begin{tabular}{|c||c|c|c|}
\hline \multirow{2}{*}{ distributions } & \multicolumn{3}{|c|}{ functions } \\
\cline { 2 - 4 } & $\phi$ & $\phi^{\prime}$ & $\frac{\phi^{\prime}}{\phi}$ \\
\hline Uniform & $\frac{1}{b-a}$ & 0 & 0 \\
\hline Exponential & $\alpha e x p(-\alpha y)$ & $-\alpha^{2} e x p(-\alpha y)$ & $-\alpha$ \\
\hline Normal & $\frac{e(y)}{\sqrt{2 \pi \sigma}}$ & $-\frac{(y-\mu) e(y)}{\sqrt{2 \pi} \sigma^{3}}$ & $-\frac{y-\mu}{\sigma^{2}}$ \\
\hline Gamma & $\frac{F(y)}{\Gamma(\nu)}$ & $\frac{F(y)(\nu-1-\alpha y)}{\Gamma(\nu) y}$ & $\frac{\nu-1}{y}-\alpha$ \\
\hline
\end{tabular}

$$
e(y) \equiv \exp \left(\frac{-(y-\mu)^{2}}{2 \sigma^{2}}\right), F(y) \equiv \alpha^{\nu} y^{\nu-1} \exp (-\alpha y), \nu \neq 1
$$

\section{Examples}

In this section, we explore condition (A) when p.d.f. $\phi(\cdot)$ is specified. If p.d.f. $\phi(\cdot)$ is uniform, exponential, normal or gamma, condition (A) can be rewritten as in Table 1 . We discuss two cases.

Case(1):

$$
\frac{\phi^{\prime}(y)}{\phi(y)} \leq \frac{h+p}{B_{2}-B_{1}} \quad \text { for all } y \in \Re^{+}, B_{2}-B_{1}>0 .
$$

If $\sup _{0<y<\infty}\left\{\frac{\phi^{\prime}(y)}{\phi(y)}\right\} \leq \frac{h+p}{B_{2}-B_{1}}$ holds, then the condition(A) is immediately satisfied. Therefore, any uniform and exponential distributions satisfy the condition (A) from Table 1.

On the other hand, if $\phi$ is a normal distribution with parameters $(\mu, \sigma)$, then condition(A) reduces to

$$
y \geq \mu-\frac{(h+p) \sigma^{2}}{B_{2}-B_{1}}>0
$$

Because $\operatorname{Pr}\{-4 \sigma+\mu<y<4 \sigma+\mu\} \approx 1$ and $\operatorname{Pr}\{0 \leq y<\infty\}=1$ in our model, the condition (A) is satisfied when

$$
4 \sigma \leq \mu \quad \text { and } \quad \frac{(h+p) \sigma}{B_{2}-B_{1}}>4
$$

If $(h+p)$ is large enough compared with $\left|B_{2}-B_{1}\right|$, then the inequality (5.3) may hold.

For a gamma distribution with parameters $(\alpha, \nu)$, condition $(\mathrm{A})$ reduces to

$$
y \geq \frac{(\nu-1)\left(B_{2}-B_{1}\right)}{\alpha\left(B_{2}-B_{1}\right)+h+p}
$$

Therefore, if

$$
0<\nu<1
$$

holds, then condition (A) holds. Furthermore, if $(5.5)$ does not hold but $(h+p)$ is large enough compared with $\left|B_{2}-B_{1}\right|$, then the inequality(5.4) may hold(see Fig. 3).

Case (2):

$$
\frac{\phi^{\prime}(y)}{\phi(y)} \geq \frac{h+p}{B_{2}-B_{1}} \quad \text { for all } y \in \Re^{+}, B_{2}-B_{1}<0
$$




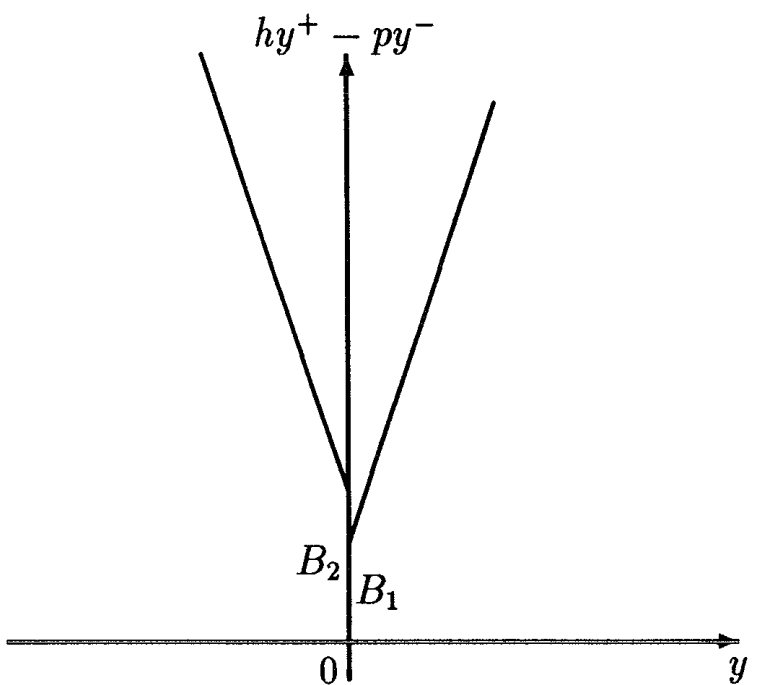

Figure 3:The Inventory costs in the case $B_{1}<B_{2}$

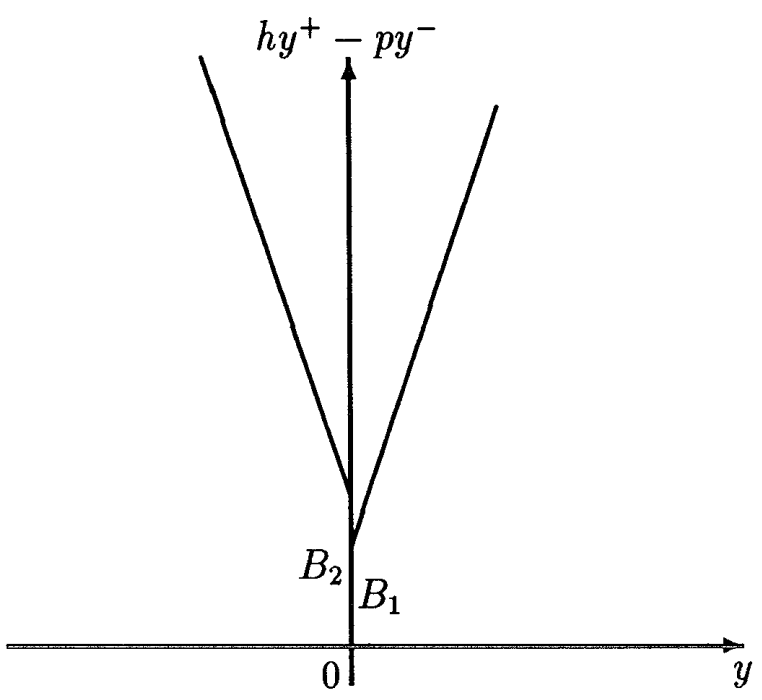

Figure 4:The Inventory costs in the case $B_{1}>B_{2}$

If $\inf _{0<y<\infty}\left\{\frac{\phi^{\prime}(y)}{\phi(y)}\right\} \geq \frac{h+p}{B_{2}-B_{1}}$ holds, then condition (A) is satisfied and hence a uniform distribution immediately satisfies condition (A).

For an exponential distribution with mean $1 / \alpha$, condition $(\mathrm{A})$ reduces to

$$
\alpha<\left|\frac{h+p}{B_{2}-B_{1}}\right|
$$

In this case, if $(h+p)$ is large enough compared with $\left|B_{2}-B_{1}\right|$, then the inequality (5.7) may hold(see Fig. 4).

For a normal distribution with parameters $(\mu, \sigma)$, condition (A) reduces to

$$
y \leq \mu-\frac{(h+p) \sigma^{2}}{B_{2}-B_{1}}(<\mu)
$$

Because $\operatorname{Pr}\{-4 \sigma+\mu<y<4 \sigma+\mu\} \approx 1$ and $\operatorname{Pr}\{0 \leq y<\infty\}=1$ in our model, condition (A) is satisfied when

$$
4 \sigma \leq \mu \quad \text { and } \quad-\frac{(h+p) \sigma}{B_{2}-B_{1}}>4
$$

If $(h+p)$ is large enough compared with $\left|B_{2}-B_{1}\right|$, then the inequality (5.9) may hold.

For a gamma distribution, condition $(\mathrm{A})$ reduces to

$$
y \leq \frac{(\nu-1)\left(B_{2}-B_{1}\right)}{\alpha\left(B_{2}-B_{1}\right)+h+p} .
$$

Therefore, if

$$
\begin{aligned}
& \alpha\left(B_{2}-B_{1}\right)+h+p<0, \\
& \left|\alpha\left(B_{2}-B_{1}\right)+h+p\right| \approx 0, \quad \text { and } \nu>1
\end{aligned}
$$

then the inequality (5.10) may hold. Summing up the above discussion, we have the following proposition which is also summarized in Table 2. 
Table 2: Examples of the results

\begin{tabular}{|c||c|c|c|c|}
\hline \multicolumn{1}{|c||}{} & \multicolumn{4}{c|}{ distributions } \\
\cline { 2 - 5 } case & Uniform & Exponential & Normal & Gamma \\
\hline$(1)$ & $\circ$ & $\circ$ & $\triangle:(5.3)$ & $\triangle:(5.4)$ \\
\hline$(2)$ & $\circ$ & $\triangle:(5.7)$ & $\triangle:(5.9)$ & $\triangle:(5.10)$ \\
\hline
\end{tabular}

$\circ$ :Condition (A) holds without any assumptions.

$\triangle:$ If the inequality in the parenthesis holds, then Condition(A) holds.

\section{Proposition 3.}

Case(1)

For any uniform distribution or exponential distribution, condition (A) holds.

If $\phi$ is a normal distribution with (5.3), or a gamma distribution with (5.4), then condition (A) holds.

Case(2)

For any uniform distribution, condition (A) holds.

If $\phi$ is an exponential distribution with (5.7), a normal distribution with (5.9), or a gamma distribution with (5.10), then condition (A) holds.

\section{Conclusion}

In this paper, we have shown under condition (A) that the $(\mathrm{s}, \mathrm{S})$ policy is optimal for finite period stochastic inventory models with fixed inventory holding and shortage costs, in addition to a fixed ordering cost. Our proof for this result is different from and much simpler than the one offered by Aneja and Noori[1]. This paper also provides an answer to the question of how robust the class of $(\mathrm{s}, \mathrm{S})$ policies is for stochastic inventory models with fixed costs .

Furthermore, we have demonstrated that condition (A) is a sufficient one for the $(\mathrm{s}, \mathrm{S})$ policy to be optimal for multi-period models. However, this condition may limit the candidates of demand functions to a certain class. When the probability density function of demand is specified such as uniform, exponential, normal or gamma, we have discussed in section 5 how condition (A) can be rewritten and whether or not it holds.

\section{Acknowledgement}

The authors would like to express our thanks to the anonymous referees for their helpful comments and suggestions which substantially improved the paper. The authors are also grateful to Professor Katsuhisa Ohno, Nagoya Institute of Technology, for his suggestions and encouragement. This research was partially supported by the Nanzan University Pache Grants for Promoting Research.

\section{References}

[1] Aneja, Y. and Noori, H. A.: The Optimality of (s,S) Policies for a Stochastic Inventory Problem with Proportional and Lump-sum Penalty Cost. Management Science, Vol. 33 (1987), 750-755.

[2] Denardo, E. V.: Dynamic Programming: Models and Applications, Prentice Hall., Inc., 1982.

[3] Lippman, S. A.: Optimal Inventory Policy with Multiple Set-up Costs. Management Science, Vol. 16 (1969), 118-138.

[4] Lippman, S. A.: Optimal Inventory Policy with Subadditive Ordering Costs and Stochastic Demands. SIAM Journal on Applied Mathematics, Vol. 17 (1969), 543-559. 
[5] Porteus, E. L.: On the Optimality of Generalized (s,S) Policies. Management Science, Vol. 17 (1971), 411-426.

[6] Ross, S. M.: Applied Probability Models with Optimization Applications, Holden-Day, San Francisco, 1970.

[7] Scarf, H.: The Optimality of (s-S) Policies in the Dynamic Inventory Problem. Chapter 13 in Arrow, K. J., Karlin, S. and Scarf, H.(Eds.), Mathematical Method in the Social Science, Stanford University Press, Stanford, 1960.

[8] Veinott, A. F., Jr. and Wagner, H. M.: The Status of Mathematical Inventory Theory. Management Science, Vol. 12 (1966), 745-777.

[9] Veinott, A. F.: Optimal Policy for a Multi-product Dynamic, Non-stationary Inventory Problems. Management Science, Vol. 12 (1965), 206-222.

[10] Veinott, A. F.: On the Optimality of $(\mathrm{s}, \mathrm{S})$ Inventory Policies: New Conditions and a New Proof. SIAM Journal on Applied Mathematics, Vol. 14 (1966), 1067-1083.

\section{Tomonori ISHIGAKI:}

Department of Systems Engineerings, Nagoya Institute of Technology, Gokiso-cho, Showa-ku Nagoya 466, Japan 